\title{
„Was für mich selbst gut ist, kann meinen Kindern nicht schaden." Der Einfluss der elterlichen Freizeitaktivitäten auf die Eltern-Kind-Aktivitäten
}

\author{
„What is good for me cannot harm my children“: The influence of \\ parental leisure activities on the home environment
}

\begin{abstract}
Zusammenfassung:
Familien variieren sehr stark darin, wie sie mit ihren Kindern umgehen. Diese Unterschiede werden oft auf die sozioökonomische Herkunft (SES) der Eltern zurückgeführt. Viele Studien können belegen, dass die Art und der Umfang des häuslichen Anregungsniveaus die Kompetenzentwicklung der Kinder stark beeinflusst. Daher stellt sich die Frage, wodurch die sozialen Unterschiede bei den Eltern-Kind-Interaktionen erklärt werden. Es wird davon ausgegangen, dass Eltern bewusst oder unbewusst im Rahmen von Aktivitäten mit ihrem Kind ihren Nutzen maximieren wollen. Diese Nutzenmaximierung zeigt sich auch in ihren eigenen hochkulturellen Freizeitaktivitäten. Somit sollten die eigenen Freizeitaktivitäten der Eltern den Umfang an häuslichen Interaktionen mit ihrem Kind vorhersagen. Dieser nutzentheoretische Ansatz wird mit den Daten des Projekts „Erwerb von sprachlichen und kulturellen Kompetenzen von Migrantenkindern in der Vorschulzeit" überprüft. Mit Hilfe von linearen Regressionsmodellen wird gezeigt, dass die sozialen Unterschiede vor allem mit dem hochkulturellen Freizeitverhalten der Eltern zusammenhängen, und nicht von der finanziellen Situation der Familien abhängen. Somit spiegeln sich die eigenen Interessen der Eltern in der häuslichen Interaktion wider, die wiederum direkt die Kompetenzen der Kinder beeinflusst.
\end{abstract}

Schlagwörter: Häusliche Aktivitäten, soziale Unterschiede, vorschulische Entwicklung, hochkulturelle Freizeitaktivitäten

\begin{abstract}
Summary:
Families differ strongly in how they deal with their children. These differences are often attributed to the socio-economic origin of the parents. Numerous studies have shown that the type and the availability of the stimuli in a child's home environment strongly influence the child's abilities. But how can the social differences in parent-child interactions be explained? It is argued that parents consciously or unconsciously want to maximize their child-related activities' utility. This maximization of utility is also visible in parents' own cultural leisure activities. Thus these should predict the extent of their interactions with their child in the home environment. This utility-theoretical approach is tested by using the data of the project "Preschool education and educational careers among migrant children". Employing linear regression models, it can be shown that the social differences are related primarily to the cultural leisure behaviour of the parents, rather than to the family's financial situation. Thus, the parents' own interests are reflected in their interaction within the home environment, which, in turn, has a direct effect upon their child's development.
\end{abstract}

Key words: Home environment, social inequality, child development, cultural leisure activities 


\section{Einleitung}

In der internationalen Forschung zur Entwicklung von Vorschulkindern gelten das familiäre Umfeld und gemeinsame Aktivitäten von Eltern und Kindern schon seit längerem als maßgebliche Ursachen für die nachhaltige Entwicklung der Kinder. So zeigen beispielsweise Luster und Dubow (1992), dass das häusliche Umfeld der Kinder (gekennzeichnet durch z.B. kognitive Stimulation oder emotionale Unterstützung durch die Eltern) einen wichtigen Einfluss auf deren Entwicklung, im speziellen auf deren verbale und kognitive Intelligenz hat. Darüber hinaus zeigt sich durchweg ein positiver Zusammenhang zwischen der Häufigkeit gemeinsamer Aktivitäten von Eltern und Kindern, wie beispielsweise dem Vorlesen aus Büchern, und dem Entwicklungsstand der Kinder (Denton/Reaney/West 2001; Melhuish et al. 2008; Raviv/Kessenich/Morrison 2004). Neuere Untersuchungen im deutschsprachigen Raum bestätigen diese internationalen Erkenntnisse (Anders et al. 2012; Biedinger 2009a; Lehrl et al. 2012; Niklas/Schneider 2012; Schöler et al. 2005; Weinert et al. 2012).

Die aktuelle Forschung zu ethnischen und sozialen Kompetenzunterschieden im frühen Lebensalter zeigt zumeist, dass die häuslichen Aktivitäten mit den Kindern (z.B. Vorlesen, gemeinsam Singen, Geschichten erzählen) eine Vermittlerrolle zwischen dem sozialen oder ethnischen Hintergrund und den Kompetenzen des Kindes einnehmen (Becker 2010a; Biedinger 2009a). Offen bleibt dabei aber die Frage nach dem dahinterliegenden Mechanismus. Wie hängt das Bildungsniveau der Eltern mit deren häuslichen Aktivitäten mit ihren sehr jungen Kindern zusammen?

Es konnte empirisch deskriptiv gezeigt werden, dass sich die Quantität und die Qualität der Eltern-Kind-Interaktion zwischen Familien stark unterscheidet (Bradley/Corwyn 2005). Dies zeigt sich besonders in der gemeinsam verbrachten Zeit (Bianchi 2000; Craig 2006; Kitterød 2002; Yeung et al. 2001), aber auch in der Art der gemeinsamen Aktivität (Lehrl et al. 2012; van Steensel 2006). Unklar bleibt jedoch, warum es soziale Unterschiede innerhalb der Eltern-Kind-Interaktion gibt. Zur Erklärung dieses Explanandums wird davon ausgegangen, dass die Eltern bewusst oder unbewusst nutzenmaximierend handeln und ihre eigenen Aktivitätsneigungen auf ihr Kind übertragen. Der positive Effekt von Bildung auf die Aktivitäten mit dem Kind sollte demnach davon abhängen, welche Aktivitäten die Eltern selbst in ihrer Freizeit ausüben (z.B. Zeitung lesen, kulturelle Aktivitäten, ehrenamtliches Engagement). Die eigene Bildung und die Freizeitaktivitäten sind zwar hoch korreliert, jedoch stehen sie bei weitem nicht in einem perfekten Zusammenhang. Die Berücksichtigung der (hochkulturellen) Freizeitaktivitäten der Eltern sollte den direkten positiven Effekt von Bildung auf die häuslichen Aktivitäten zumindest verringern, eventuell sogar komplett verschwinden lassen. Somit sollten die elterlichen Freizeitaktivitäten einen entscheidenden Mediator zur Erklärung von sozialen Unterschieden in den häuslichen Aktivitäten darstellen.

Daher wird im Folgenden zunächst der Forschungsstand zu den Determinanten von häuslichen Eltern-Kind-Aktivitäten vorgestellt (Abschnitt 2). Danach wird in Abschnitt 3 theoretisch herausgearbeitet, warum die häuslichen Aktivitäten überhaupt einen zentralen Einfluss auf die Entwicklung des Kindes ausüben sollten und worin der Zusammenhang zwischen sozialer Herkunft, hochkulturellen Freizeitaktivitäten der Eltern und der ElternKind-Interaktion bestehen. Im Anschluss daran werden in Abschnitt 4 die Daten vorgestellt. Die letzten beiden Abschnitte stellen die Ergebnisse vor (Abschnitt 5) und diskutieren diese (Abschnitt 6). 


\section{Forschungsstand zu den Determinanten von häuslichen Aktivitäten}

In dem vorliegenden Beitrag werden die häuslichen Aktivitäten mit dem Kind relativ weit definiert. Im internationalen Kontext wird dies zumeist mit der HOME-Scale gemessen (vgl. Totsika/Sylva 2004). Zur Erklärung von Sprachfähigkeiten wird die anregungsreiche häusliche Umgebung meist als HLE (Home Literacy Environment) bezeichnet (vgl. Niklas/ Schneider 2013; Sénéchal/Young 2008; ein Überblick über die verschiedenen Formen findet sich bei Niklas/Schneider 2010). Unter die häuslichen Aktivitäten fasst der vorliegende Beitrag jegliche gemeinsame Zeit, die zur Förderung des Kindes beiträgt, unabhängig davon, ob damit die sprachlichen oder kognitiven Fähigkeiten gefördert werden.

Das Ausmaß, in dem häusliche Aktivitäten in den Familien stattfinden, hängt von verschiedenen Faktoren ab, die im Folgenden vorgestellt werden sollen. Da in vielen Studien nicht die Qualität der Aktivitäten, sondern eher die Quantität (Verwendung von Zeittagebuch-Daten (Künzler et al. 2001; Walter/Künzler 2002; Zick/Bryant/Österbacka 2001)) unterschieden wird, werden in dieser Arbeit beide Forschungsrichtungen berücksichtigt. Es wird jedoch bewusst auf primäre Betreuungszeit fokussiert, die das Hauptaugenmerk auf die anregende Betreuung und nicht auf die Pflege legt.

In der internationalen Literatur zeigt sich im Allgemeinen das Alter des jüngsten Kindes als eine wichtige Determinante für die Zeit, die Eltern in primärer Betreuungszeit verbringen (Bryant/Zick 1996a; 1996b; Hill/Stafford 1980; Kitterød 2002; Walter/Künzler 2002; Zick/Bryant 1996). So kommen Künzler et al. (2001) bei ihren Analysen in Deutschland zu dem Ergebnis, dass Mütter, deren Kinder unter sechs Jahre alt sind, ca. fünfeinhalb Stunden pro Woche mehr mit der Kinderbetreuung beschäftigt sind, als sie es mit Kindern im Alter zwischen sieben und 12 Jahren sind. Bei Vätern sind es etwas weniger als vier Stunden. Auch die Qualität und der Umfang der Stimulation in der Eltern-Kind-Interaktion werden maßgeblich durch das Alter des Kindes begründet (z.B. Yarosz/ Barnett 2001).

Eine weitere wichtige Determinante für die elterliche Betreuungszeit ist die Bildung der Eltern. Empirisch zeigt sich ein positiver Zusammenhang zwischen der Bildung der Eltern und deren absoluter Betreuungszeit (Bianchi 2000; Craig 2006; Hill/Stafford 1980; Kitterød 2002; Künzler et al. 2001; Sandberg/Hofferth 2001; Walter/Künzler 2002; Yeung et al. 2001). Mit der Bildung der Betreuungsperson hängt auch deren Erwerbstätigkeit zusammen. Der Einfluss, den die Erwerbstätigkeit (meist der Mütter) auf die Kinderbetreuung ausübt, ist nicht von vornherein eindeutig. Es erscheint logisch, dass eine erwerbstätige Mutter nicht genauso viel Zeit mit ihren Kindern verbringen kann, wie eine nicht erwerbstätige Mutter. Alleine durch die Arbeitszeit verbringen erwerbstätige Mütter zwangsläufig weniger Zeit im eigenen Haushalt. Es hat sich jedoch in mehreren Studien gezeigt, dass das Austauschverhältnis von mütterlicher Arbeitszeit und elterlicher Kinderbetreuungszeit geringer ist als eins. Dies bedeutet, dass die elterliche Betreuungszeit um weniger als eine Stunde pro Arbeitsstunde der Mutter sinkt (Bianchi 2000; Bryant/ Zick 1996b; Craig 2007; Künzler et al. 2001; Sandberg/Hofferth 2001). Eine mögliche Erklärung hierfür ist eine Erhöhung der väterlichen Betreuungszeit, welche eine sinkende mütterliche Betreuungszeit kompensiert. Diese theoretischen Überlegungen können jedoch empirisch nicht belegt werden (Hofferth 2001). Wie Craig (2007) zeigt, kommt es eher durch Reorganisation mütterlicher Aktivitäten sowie einer Reduktion der Zeit, die Mütter mit Hausarbeit und persönlicher Freizeit verbringen, zu diesem niedrigen Aus- 
tauschverhältnis. In zwei Studien zeigt sich sogar, dass gerade die Zeit, die in entwicklungsfördernden Aktivitäten mit den Kindern verbracht wird, nicht verringert wird (Hofferth 2001; Zick/Bryant/Österbacka 2001). Mitunter findet sich sogar ein positiver Zusammenhang zwischen der Erwerbstätigkeit der Mutter und der Häufigkeit bestimmter entwicklungsfördernder Aktivitäten, wie beispielsweise den Kindern vorzulesen oder älteren Kindern bei den Hausaufgaben zu helfen (Zick/Bryant/Österbacka 2001). Fasst man die beiden letzten Variablen zum sozio-ökonomischen Status zusammen, so können Studien belegen, dass auch unter Kontrolle anderer Variablen dieser einen großen Teil der Variation der häuslichen Umwelt erklären kann (vgl. Bradley et al. 1994; Garrett/ Ng'andu/Ferron 1994; Hoff 2003). Wenigstens eine Studie kommt zu dem Ergebnis, dass der Unterschied im Verhalten der Mütter in der Interaktion mit ihrem Kind auf Basis ihrer sozialen Herkunft schon recht früh im Alter von 6 bis 20 Monaten belegt werden kann (vgl. Farran/Ramey 1980). Auf der anderen Seite ist es denkbar, dass die ökonomische Situation ebenfalls einen starken Einfluss auf die anregenden Aktivitäten ausübt, da finanzielle Engpässe mit anderen Arten von Aktivitäten einhergehen können. Diese Vermutung können Hashima und Amato (1994) jedoch nicht bestätigen. Das Freizeitverhalten der Eltern hat - unter Kontrolle des Einkommens - in dieser Studie keine Auswirkungen auf das Verhalten mit dem Kind (weder auf bestrafendes noch unterstützendes Verhalten).

In Bezug auf das Geschlecht der Kinder zeigt sich in bisherigen Studien, dass es eine geschlechtsspezifische Betreuung gibt: Mütter verbringen demnach mehr Betreuungszeit mit Töchtern als mit Söhnen, bei Vätern verhält es sich umgekehrt (Bryant/Zick 1996a; Walter/Künzler 2002). Zick und Bryant (1996) vermuten die Gründe hierfür einerseits auf Elternseite, andererseits auf Kinderseite. So könnten die Eltern, nach Ansicht der Autoren, die Möglichkeit, geschlechtsrollenspezifische Sozialisation zu fördern, zu schätzen wissen. Andererseits sei es auch denkbar, dass Kinder bewusst Interaktionen mit dem gleichgeschlechtlichen Elternteil suchen.

Zusammenfassend lassen sich also das Alter des jüngsten Kindes, das Geschlecht des Kindes, die Erwerbstätigkeit der Eltern, sowie die Bildung der Eltern als wichtige Determinanten für die Quantität und Qualität der Eltern-Kind-Interaktion festhalten. Vor allem die Bildung beeinflusst die gemeinsamen Aktivitäten stark, auch wenn deren Wirkungsrichtung noch kontrovers diskutiert wird. Hierzu fehlt es in den bisherigen Studien auch an einem theoretischen Erklärungsansatz, der Licht in das Dunkel bringen könnte. Der folgende Absatz versucht diese Lücke im theoretischen Modell zu schließen.

\section{Theoretischer Hintergrund}

\subsection{Modell des frühen Kompetenzerwerbs}

Die häuslichen Aktivitäten zwischen Eltern und ihren Kindern spielen bei der frühen Kompetenzentwicklung eine entscheidende Rolle. Das Modell des frühen Kompetenzerwerbs leitet sich aus verschiedenen Forschungstraditionen ab. Es vereint Annahmen aus der Bildungsforschung (Boudon 1974; Bourdieu 1976; Lareau 2002), der schichtspezifischen Sozialisationsforschung (Kohn 1969), der Entwicklungspsychologie (Bandura 1977; 
Conger/Rueter/Conger 2000) und aus ökonomischen Ansätzen (Becker/Tomes 1976; für einen Überblick der Theorien: siehe Biedinger 2009a). Im Zentrum dieses Modells steht der frühe Erwerb von Kompetenzen. Es kann zwischen verschiedenen Kompetenzen unterschieden werden (z.B. sprachliche, kulturelle, kognitive), die für die Schulfähigkeit, für den Start der Bildungslaufbahn und für die langfristige Bildungskarriere von großer Bedeutung sind. Gerade für sehr junge Kinder kann angenommen werden, dass die Kompetenzen vor allem durch die Familie und durch altersspezifische Einflussfaktoren beeinflusst werden (Becker/Biedinger 2006; Biedinger/Becker 2010).

Die Familie ist der Ausgangspunkt des theoretischen Erklärungsmodells. Ein großer Teil der Förderung der Kinder findet im Rahmen der Sozialisation innerhalb der Familien statt (Grundmann 2001, Lehrl et al. 2012). Die Familie kann die Kompetenzen des Kindes sowohl direkt beeinflussen, als auch indirekt über die häuslichen Aktivitäten (z.B. ein förderliches häusliches Umfeld mit stimulierenden Spielsachen etc.) oder durch Entscheidungen bezüglich des Kindergartens und anderen vorschulischen Institutionen, die das Kind besuchen kann. Dabei unterscheiden sich die Familien in vielerlei Hinsicht, wie z.B. in der Schichtzugehörigkeit, im Bildungsstand, im Einkommen, in der kulturellen Herkunft oder auch der Erwerbstätigkeit.

Zusammenfassend zeigt sich, dass die Auswirkungen des Vorschulbesuchs und der häuslichen Aktivitäten der Eltern auf die kindliche Entwicklung international gut belegt sind (z.B. Bus et al. 1995; Downer/Pianta 2006; Grundmann 2001; Hart/Risley 1995). In jüngster Zeit konnten an verschiedenen Stellen auch für Deutschland klare Zusammenhänge zwischen den häuslichen Aktivitäten und verschiedenen Kompetenzmaßen festgestellt werden (Anders et al. 2012; Becker 2010a, 2010b; Biedinger 2009a, 2009b; Lehrl et al. 2012; Niklas/Schneider 2012; Schöler et al. 2005; Weinert et al. 2012). Offen bleibt jedoch bei diesen Analysen, warum sich Familien unterschiedlicher sozialer oder ethnischer Herkunft in ihren häuslichen Aktivitäten unterscheiden.

\subsection{Theoretische Erklärungen von unterschiedlichen Häufigkeiten der Eltern- Kind-Aktivitäten}

Die dargestellten empirischen Befunde können bisher keine Erklärung dafür liefern, warum sich Eltern in Bezug auf ihren sozialen Hintergrund im Umgang mit ihren Kindern unterscheiden. Somit besteht die Aufgabe darin, das soziale Handeln der Eltern zu erklären. Daran knüpft die Frage an, ob es sich bei den Eltern-Kind-Aktivitäten um zweckrationales, wertrationales, affektuelles oder traditionales Handeln im Weberschen Sinne handelt (Weber 1980), oder ob sie verwandte Typen des Handelns sind, wie beispielsweise normatives oder habituelles Handeln (siehe dazu auch weiter das Modell der Frame-Selektion z.B. bei Kroneberg 2007). Unabhängig vom tatsächlichen Typ des Handelns, wird jedoch davon ausgegangen, dass die Basis der Handlungsentscheidung auf einer Nutzenabwägung beruht, die durch den Handlungstyp bestimmt ist. Demnach sollte bei einem wertrationalen Handeln der Nutzen durch den eingeschätzten Wert der Handlung so hoch sein, dass die Handlung vollzogen wird. Ein anderes Beispiel wäre das traditionnelle Handeln, bei dem die Einhaltung einer Tradition als so nützlich empfunden wird, so dass auch dieser Handlungstyp im Endeffekt auf einer Kosten-Nutzen-Abwägung 
beruht. Eine nutzenbasierte Modellierung sollte Aufschluss darüber geben, warum bestimmte soziale Gruppen sich im Ausmaß ihrer Aktivitäten unterscheiden. Die KostenNutzen-Abwägung der Eltern spielt also demnach eine entscheidende Rolle, wobei diese subjektive Einschätzung des Nutzens und ggf. auch der Kosten, durch Werte, Traditionen und andere Einflussfaktoren bestimmt sein kann.

Das Hauptaugenmerk liegt auf der Erklärung der sozialen Unterschiede. Somit müsste die Definition der Situation für Eltern von deren formaler Bildung abhängen. Diese Annahme ist eher fragwürdig, da die Eltern unabhängig von ihrer Schulbildung über zahlreiche (erziehungsrelevante) Erfahrungen verfügen können, so dass die Basis der Situationsdefinition eher in Erfahrungen zu suchen ist, welche allerdings durch die Bildung selektiv sein könnten (zur Definition der Situation siehe Esser 1996). So wurde beispielswiese an verschiedenen Stellen gezeigt, dass sich das kulturelle Kapital, gemessen durch spezifische Freizeitaktivitäten, zwischen Personen unterschiedlicher sozialer Herkunft stark unterscheidet (vgl. Diewald/Schupp 2004; Rössel/Beckert-Zieglschmid 2002). Dennoch sind auch hochkulturelle Freizeitaktivitäten nicht perfekt mit der sozialen Herkunft korreliert. Rössel und Beckert-Zieglschmid (2002) zeigen, dass nur 35 Prozent der Variation im hochkulturellen Kapital auf Variablen der Bildung und der beruflichen Stellung zurückgeführt werden können. Somit könnte es sich bei den häuslichen Aktivitäten eher um habitualisiertes, traditionelles oder geframtes Handeln der Eltern handeln. Daraus lässt sich die Vermutung ableiten, dass sich Eltern aufgrund der eigenen Freizeitaktivitäten und eben nicht aufgrund des eigenen Bildungsniveaus - darin unterscheiden in welchem Umfang sie häusliche Aktivitäten mit ihren Kindern durchführen. Somit sollten die Freizeitaktivitäten der Eltern einen bedeutsamen Mediator zwischen der Bildung und den Aktivitäten mit dem Kind darstellen. Dieser Zusammenhang zwischen den eigenen Freizeitaktivitäten und den häuslichen Aktivitäten wird gewiss auch durch die Normvorstellungen, Werte oder Traditionen der Eltern beeinflusst, da sich diese ebenfalls in deren eigenen widerspiegeln sollten.

Um die Relevanz der Fragestellung zu demonstrieren wird in einem allerersten Schritt mit den vorliegenden Daten auch gezeigt, dass die häuslichen Aktivitäten von zentraler Bedeutung für die Kompetenzen der Kinder sind. Im Anschluss daran leiten sich drei Fragestellungen ab, die der vorliegende Artikel beantworten möchte.

Erstens ist davon auszugehen, dass höher gebildete Eltern auch häufiger entwicklungsfördernde Aktivitäten mit ihren Kindern durchführen. Die Frage ist demnach, wie sich die Bildung der Eltern auf die gemeinsamen Aktivitäten auswirkt. Zweitens sollte geprüft werden, ob dieser Zusammenhang nicht durch andere Drittvariablen (vor allem das Einkommen) hervorgerufen wird, da beispielsweise finanzielle Einschränkungen die Möglichkeit zu kostenintensiven Aktivitäten begrenzen. Daran schließt sich drittens die Hauptfragestellung des Papiers an, nämlich ob das elterliche (hochkulturelle) Freizeitverhalten mit den Eltern-Kind-Aktivitäten zusammenhängt und darüber hinaus, ob die Freizeitaktivitäten den Einfluss der Bildung vermitteln können. Ausgehend von den theoretischen Überlegungen, sollten häufigere elterliche Freizeitaktivitäten dazu führen, dass auch die Eltern-Kind-Aktivitäten zunehmen. Zusätzlich wird davon ausgegangen, dass die Bildung an sich nicht die Eltern-Kind-Aktivitäten beeinflusst, sondern nur indirekt über das Freizeitverhalten der Eltern wirkt. Hier liegt die Annahme zu Grunde, dass die eigenen Freizeitaktivitäten näherungsweise den von den Eltern wahrgenommenen Nutzen beschreibt. 


\section{Datensatz und Operationalisierung}

Die folgenden empirischen Analysen basieren auf einem Datensatz, der im Rahmen des von der DFG geförderten Projekts „Erwerb von sprachlichen und kullturellen Kompetenzen von Migrantenkindern in der Vorschulzeit" an der Universität Mannheim entstanden ist. Es handelt sich um die deutsche Stichprobe ${ }^{1}$ aus der zweiten Welle der Längsschnittstudie, die im Frühjahr 2008 durchgeführt wurde. Insgesamt wurden dabei 579 deutsche Familien aus 30 Städten/Gemeinden im Großraum Rhein-Neckar befragt und die Fähigkeiten ihrer Kinder getestet. Diese Tests wurden im Anschluss an die Befragung der Eltern durch den psychologischen Entwicklungstest „Kaufman-Assessment Battery for Children“ (K-ABC) in der deutschsprachigen Fassung mit den vier- bis fünfjährigen Kindern durchgeführt (Kaufman/Kaufman 1994; deutschsprachige Fassung: Melchers/Preuß 2001). Die K-ABC ist ein Individualtest zur Messung von Fertigkeiten bei Kindern im Alter von 2;6 (zwei Jahre und sechs Monate) bis 12;5 Jahren, wobei jeweils altersgerechte Aufgaben bearbeitet werden müssen. Die folgenden Analysen werden mit 488 Fällen durchgeführt, da Fälle mit fehlenden Werten auf den Modellvariablen von den Analysen ausgeschlossen wurden. Die einzelnen Variablen sind folgendermaßen operationalisiert:

\section{Kognitive Kompetenzen (abhängige Variable):}

Im Rahmen der K-ABC wurden verschiedene Untertests durchgeführt, die die ersten kognitiven Fähigkeiten der Kinder messen. Melchers und Preuß (2003) unterscheiden dabei ganzheitliches und einzelheitliches Denken, welches sich unter der Skala der intellektuellen Fähigkeiten zusammenfassen lässt: Jede Aufgabe des einzelheitlichen Denkens umfasst ein Problem, das durch folgerichtiges oder serielles Denken gelöst werden muss (z.B. Zahlennachsprechen). Bei Aufgaben des ganzheitlichen Denkens ist das Problem räumlichgestalthaft, verlangt Analogieschlüsse oder eine Organisation der Reize (z.B. Zauberfenster). Laut Pauen, Pahnke und Valentiner (2007) messen die Untertests der K-ABC sowohl fluide als auch kristalline Intelligenz, wobei das von mir als kognitive Kompetenzen bezeichnete Konstrukt vor allem die fluide Intelligenz umfasst. Dagegen werden die sprachlichen Kompetenzen als kristalline Intelligenz bezeichnet. Zur Veranschaulichung der zentralen Auswirkungen von häuslichen Aktivitäten auf die kognitiven Kompetenzen der Kinder, wird ein Faktorscore gebildet (Eigenwert 1,83). Dieser setzt sich aus vier Untertests zusammen, von denen jeweils die Summe der richtigen Antworten verwendet wird. Folgende Untertests werden berücksichtigt (Faktorladungen (a) in Klammern):

Zauberfenster: Benennung eines Bildes, das nur durch einen kleinen Schlitz zu sehen ist $(\mathrm{a}=0,77)$

Wiedererkennen von Gesichtern: Wiedererkennen eines Gesichts aus einem Gruppenbild $(\mathrm{a}=0,61)$;

Gestaltschließen: Benennung eines unvollständig dargebotenen „Tintenklecks-Bildes“ $(\mathrm{a}=0,69)$;

1 Das Projekt wurde bei deutschen Familien und Familien mit türkischem Migrationshintergrund durchgeführt. 
Zahlennachsprechen: Nachsprechen einer Zahlenfolge

$(\mathrm{a}=0,61)$.

Sprachliche Kompetenzen (abhängige Variable):

Das Maß der sprachlichen Kompetenzen wird durch einen einzelnen Untertest gemessen, der den Wortschatz des Kindes erfasst (Wertebereich: 0-24). Diese Variable wird für die späteren Analysen z-standardisiert.

Häusliche Aktivitäten (abhängige und unabhängige Variable):

Die Eltern wurden gefragt:

„Kinder und Familien unternehmen viele verschiedene Aktivitäten. Ich möchte wissen, ob in Ihrer Familie die folgenden Aktivitäten jeden Tag, mehrmals pro Woche, einmal pro Woche, mehrmals im Monat, einmal pro Monat, seltener oder nie durchgeführt werden."

Ich habe einen Faktor (Eigenwert 1,45) aus folgenden Angaben zur Häufigkeit (1 (nie) bis 7 (täglich)) dieser Aktivitäten gebildet: Puzzeln $(\mathrm{a}=0,49)$, Spielen $(\mathrm{a}=0,44)$, aus Büchern vorlesen $(a=0,60)$, Geschichten erzählen $(a=0,68)$ und Lieder singen $(a=0,53){ }^{2}$

Soziale Herkunft (unabhängige Variable):

Die soziale Herkunft wird über den Bildungsabschluss des hauptsächlich für die Betreuung zuständigen Elternteils operationalisiert. Es wird zwischen Hauptschule, Realschule und (Fach-)Abitur unterschieden.

Finanzielle Möglichkeiten (unabhängige Variable):

Die finanziellen Möglichkeiten der Familien werden über das Haushaltsnettoäquivalenzeinkommen gemessen. Da es bei der Frage nach den Einkommensverhältnissen relativ viele Verweigerungen gibt, wird in den multivariaten Analysen eine Dummyvariable ergänzt, die angibt, ob der Befragte diese Information verweigert hat.

Freizeitaktivitäten der Eltern (unabhängige Variable):

Faktor (Eigenwert 1,88) aus den Angaben der Eltern zur Häufigkeit von folgenden Freizeitaktivitäten ( 1 (nie) bis 6 (täglich)): Bücher lesen ( $\mathrm{a}=0,66)$, Zeitung lesen $(\mathrm{a}=0,36)$, ehrenamtliche Tätigkeiten $(\mathrm{a}=0,59)$, künstlerische/musische Tätigkeiten $(\mathrm{a}=0,65)$ und $\mathrm{Be}$ such von Opern/klassischen Konzerten $(a=0,73)$.

2 Insgesamt sind diese Aktivitäten relativ kostengünstig, jedoch bei weitem nicht kostenfrei (z.B. Anschaffung von Büchern und Spielen). 
Weitere unabhängige Variablen:

Erwerbstätigkeit der Betreuungsperson, Alter des Kindes in Monaten, Geschlecht des Kindes, Kindergartenbesuchsdauer in Stunden seit Beginn des Kindergartens, Anzahl der Kinder im Haushalt und Anzahl Kinderbücher im Haushalt.

\section{Ergebnisse}

Tabelle 1 gibt einen deskriptiven Überblick über die Verteilung der einzelnen Variablen. Spalte 1 zeigt die Mittelwerte der Gesamtstichprobe, die folgenden Spalten zeigen jeweils die Mittelwerte für Betreuungspersonen, die einen Hauptschulabschluss, Realschulabschluss oder das (Fach-)Abitur gemacht haben.

Tabelle 1: Deskriptive Statistik der Modellvariablen (Mittelwerte und Standardabweichungen bzw. Anteilswerte)

\begin{tabular}{|c|c|c|c|c|c|c|c|c|}
\hline \multirow[b]{2}{*}{ Kognitive Fähigkeiten } & \multicolumn{2}{|c|}{ Gesamt } & \multicolumn{2}{|c|}{ Hauptschule } & \multicolumn{2}{|c|}{ Realschule } & \multicolumn{2}{|c|}{ Gymnasium } \\
\hline & 0 & $(1,00)$ & $-0,13$ & $(1,08)$ & $-0,12$ & $(0,95)$ & 0,11 & $(1,00)$ \\
\hline Zauberfenster (0-15) & 8,82 & $(2,61)$ & 8,05 & $(2,83)$ & 8,63 & $(2,70)$ & 9,10 & $(2,46)$ \\
\hline Wiederkennen v. Gesichtern (0-15) & 7,03 & $(2,87)$ & 7,28 & $(3,20)$ & 7,02 & $(2,84)$ & 6,98 & $(2,82)$ \\
\hline Gestaltschließen (0-15) & 6,23 & $(2,94)$ & 6,02 & $(3,09)$ & 6,06 & $(2,69)$ & 6,37 & $(3,04)$ \\
\hline Zahlen nachsprechen $(0-12)$ & 5,15 & $(2,28)$ & 5,05 & $(1,91)$ & 4,65 & $(2,02)$ & 5,45 & $(2,44)$ \\
\hline Sprachliche Fähigkeiten (0-24) & 18,37 & $(2,68)$ & 17,70 & $(2,63)$ & 17,98 & $(2,82)$ & 18,72 & $(2,55)$ \\
\hline Aktivitäten (1-7) & 0 & $(1,00)$ & $-0,32$ & $(1,43)$ & 0,01 & $(0,93)$ & 0,07 & $(0,91)$ \\
\hline Geschichten erzählen & 5,83 & $(1,61)$ & 5,46 & $(1,85)$ & 5,84 & $(1,55)$ & 5,90 & $(1,58)$ \\
\hline Gesellschaftsspiele & 5,46 & $(1,21)$ & 5,76 & $(1,42)$ & 5,52 & $(1,06)$ & 5,36 & $(1,24)$ \\
\hline Lieder singen & 5,79 & $(1,32)$ & 5,49 & $(1,59)$ & 5,67 & $(1,48)$ & 5,93 & $(1,14)$ \\
\hline Vorlesen & 6,62 & $(0,74)$ & 6,03 & $(1,38)$ & 6,58 & $(0,66)$ & 6,77 & $(0,47)$ \\
\hline Puzzeln & 5,10 & $(1,47)$ & 5,49 & $(1,47)$ & 5,29 & $(1,35)$ & 4,91 & $(1,51)$ \\
\hline \multicolumn{9}{|l|}{ Bildung (Anteilswerte) } \\
\hline Hauptschule & 0,12 & & & & & & & \\
\hline Realschule & 0,32 & & & & & & & \\
\hline (Fach-)Abitur & 0,56 & & & & & & & \\
\hline Elterliche Freizeitaktivitäten (stand.) & 0 & $(1,00)$ & $-0,76$ & $(1,09)$ & $-0,19$ & $(0,96)$ & 0,27 & $(0,85)$ \\
\hline Alter in Monaten & 54,62 & $(3,99)$ & 55,08 & $(3,83)$ & 54,29 & $(3,86)$ & 54,70 & $(4,09)$ \\
\hline Junge & 0,49 & & 0,42 & & 0,45 & & 0,53 & \\
\hline Äquivalenzeinkommen/1000 & 1674 & $(1143)$ & 1255 & $(532)$ & 1479 & $(567)$ & 1874 & $(1410)$ \\
\hline Vollzeit Erwerbstätigkeit & 0,46 & & 0,32 & & 0,48 & & 0,48 & \\
\hline Anzahl Kinder im Haushalt & 2,18 & $(0,86)$ & 2,24 & $(1,07)$ & 2,11 & $(0,86)$ & 2,21 & $(0,81)$ \\
\hline Anzahl Kinderbücher & 74 & $(74)$ & 45 & $(41)$ & 67 & $(85)$ & 84 & (71) \\
\hline Kindergartenbesuchsdauer Stunden & 878 & 799 & 1007 & 881 & 839 & 821 & 872 & 768 \\
\hline $\mathrm{N}$ & \multicolumn{2}{|c|}{486} & \multicolumn{2}{|c|}{59} & \multicolumn{2}{|c|}{154} & \multicolumn{2}{|c|}{273} \\
\hline
\end{tabular}

Quelle: Projekt „Erwerb von sprachlichen und kulturellen Kompetenzen von Migrantenkindern in der Vorschulzeit" (Welle 2), eigene Berechnungen.

Ziel des Beitrags ist es, Unterschiede in den häuslichen Aktivitäten zu erklären. Doch zuvor wird der bedeutende Einfluss der häuslichen Aktivitäten auf die kindlichen Kompe- 
tenzen dargestellt und es wird gezeigt, dass sich Familien signifikant darin unterscheiden, welche Aktivitäten sie mit ihren Kindern durchführen. Deshalb präsentiert Tabelle 2 die Ergebnisse multivariater Analysen (OLS-Regressionen). Zunächst zeigt Tabelle 2, dass die häuslichen Aktivitäten einen signifikanten Einfluss auf die kognitiven Fähigkeiten des Kindes ausüben, auch unter Kontrolle der kindspezifischen Merkmale, der sozialen Herkunft und der Kindergartenbesuchsdauer. Neben dem Alter des Kindes und dem Einkommen spielen die häuslichen Aktivitäten die wichtigste Rolle bei der Erklärung der kognitiven Unterschiede.

Tabelle 2: Einfluss von häuslichen Aktivitäten auf die kognitiven und sprachlichen Fähigkeiten des Kindes

\begin{tabular}{|c|c|c|c|c|}
\hline \multirow[b]{2}{*}{ Häusliche Aktivitäten } & \multicolumn{2}{|c|}{ Kognitive F. } & \multicolumn{2}{|c|}{ Sprachliche F. } \\
\hline & 0,10 & $(0,05)^{\star}$ & 0,05 & $(0,05)$ \\
\hline \multicolumn{5}{|l|}{ Bildung $^{a}$} \\
\hline Realschule & 0,04 & $(0,14)$ & 0,06 & $(0,15)$ \\
\hline (Fach-)Abitur & 0,10 & $(0,13)$ & 0,18 & $(0,14)^{*}$ \\
\hline Alter Zielkind in Monaten & 0,49 & $(0,01)^{\star * *}$ & 0,32 & $(0,01)^{\star * *}$ \\
\hline Geschlecht $^{b}$ & 0,01 & $(0,08)$ & 0,08 & $(0,09)+$ \\
\hline Äquivalenzeinkommen & 0,09 & $(0,03)^{\star \star}$ & 0,04 & $(0,03)$ \\
\hline Missingvariable: Einkommen & $-0,01$ & $(0,15)$ & 0,02 & $(0,12)$ \\
\hline Erwerbstätigkeit $^{\mathrm{C}}$ & 0,05 & $(0,09)$ & 0,01 & $(0,09)$ \\
\hline Anzahl Kinder im Haushalt & $-0,04$ & $(0,05)$ & $-0,10$ & $(0,06)+$ \\
\hline Anzahl Kinderbücher/100 & 0,01 & $(0,05)$ & 0,04 & $(0,06)$ \\
\hline Kindergartenbesuchsdauer & $-0,05$ & $(0,00)$ & 0,00 & $(0,00)$ \\
\hline Elterliche Freizeitaktivitäten & $-0,03$ & $(0,05)$ & $-0,04$ & $(0,05)$ \\
\hline Konstante & $-6,84$ & $(0,63)^{\star \star *}$ & $-4,65$ & $(0,65)^{\star \star *}$ \\
\hline $\mathrm{N}$ & \multicolumn{2}{|c|}{486} & \multicolumn{2}{|c|}{477} \\
\hline $\mathrm{R}^{2}$ & \multicolumn{2}{|c|}{0,2552} & \multicolumn{2}{|c|}{0,1572} \\
\hline
\end{tabular}

Anmerkung: Standardisierte Regressionskoeffizienten mit robusten Standardfehlern (in Klammern).

Referenzkategorien: a) Hauptschule, b) Mädchen, c) nicht Vollzeit erwerbstätig.

Signifikanzniveau: $+p \leq 0,10 ; * \mathrm{p} \leq 0,05 ; * * \mathrm{p} \leq 0,01 ; * * * \mathrm{p} \leq 0,001$.

Quelle: Projekt „Erwerb von sprachlichen und kulturellen Kompetenzen von Migrantenkindern in der Vorschulzeit“ (Welle 2), eigene Berechnungen.

Die sprachlichen Fähigkeiten des Kindes (2. Spalte) werden vor allem durch das Alter des Kindes und durch die soziale Herkunft erklärt. Die häuslichen Aktivitäten haben auf diese abhängige Variable keinen signifikanten Einfluss, der Tendenz nach ist jedoch auch hier ein positiver Effekt zu erkennen. Dieser zunächst überraschende Befund ist dadurch zu erklären, dass die sprachlichen Fähigkeiten (hier operationalisiert durch den Wortschatz) vor allem durch das Vorlesen verbessert werden und weniger durch die anderen Aktivitäten, bei denen die Eltern ihr eigenes Sprachniveau höchstwahrscheinlich stark dem Kind anpassen. Insgesamt lässt sich also mit dem vorliegenden Datensatz nachweisen, dass auch unter Kontrolle von kindspezifischen und anderen familiären Variablen der signifikante Einfluss der häuslichen Aktivitäten zumindest auf die kognitiven Fähigkeiten bestehen bleibt.

Die Familien unterscheiden sich jedoch darin, wie häufig sie bestimmte Aktivitäten ausüben. Dies veranschaulicht Abbildung 3. 
Abbildung 3: Verteilung der häuslichen Aktivitäten bei Familien, bei denen die Betreuungsperson den Hauptschulabschluss ( $\mathrm{N}=59$ ), den

Realschulabschluss (N=154) oder das (Fach-)Abitur $(\mathrm{N}=273)$ besitzt

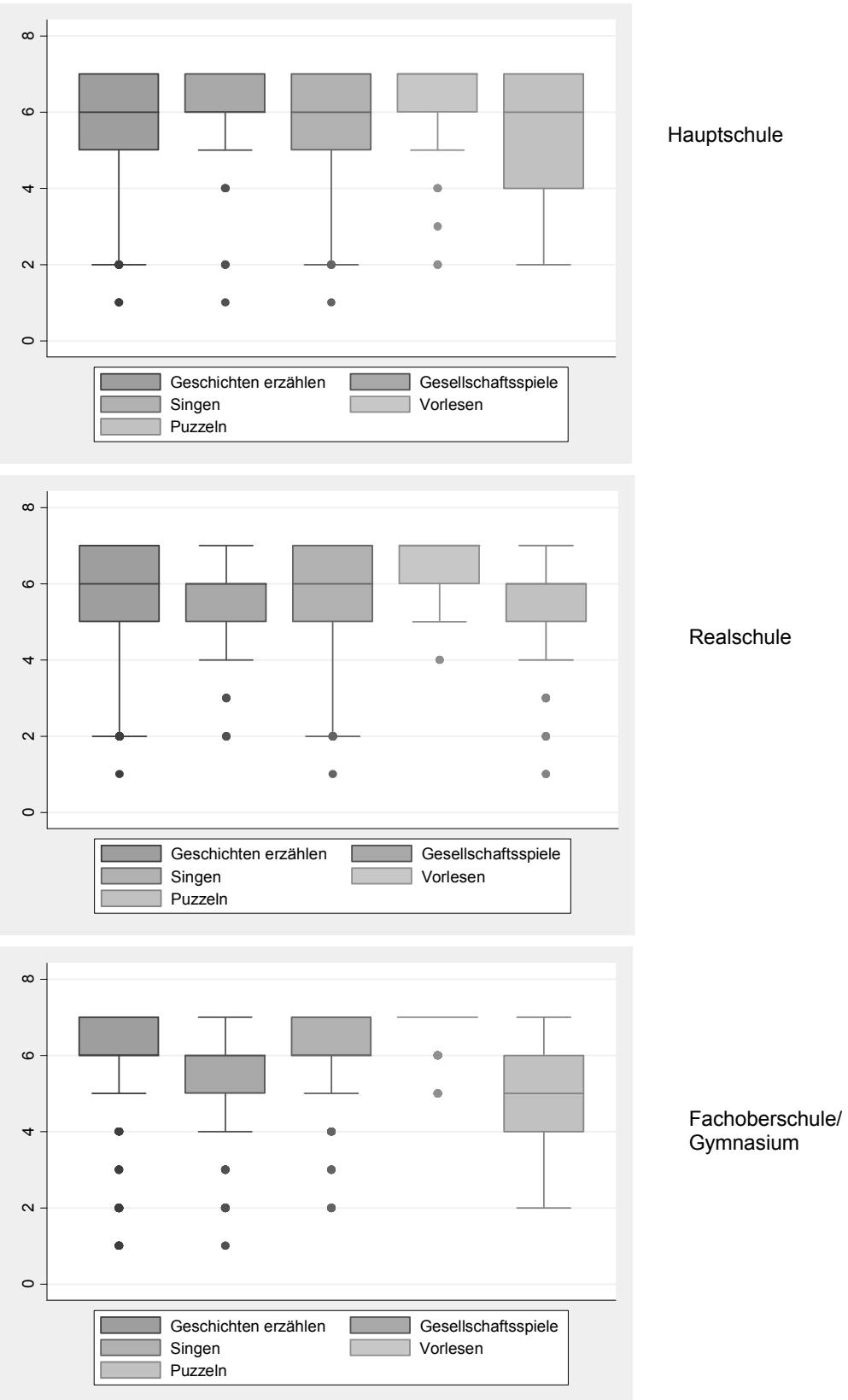

Anmerkungen zur Skala: 1=nie, 2=seltener, 3=1mal/Monat, 4=mehrmals/Monat, 5=1mal/Woche, $6=$ mehrmals pro Woche, $7=$ täglich 
Dabei wird für verschiedene Aktivitäten, nämlich Geschichten erzählen, Singen, Puzzeln, Vorlesen und das Spielen von Brettspielen gezeigt, wie sich die Antworten zwischen Familien mit unterschiedlichem Bildungshintergrund unterscheiden. Die Aktivitäten werden bei Familien mit Hauptschulabschluss insgesamt recht häufig ausgeführt, so dass alle Aktivitäten von der Mehrzahl der Befragten (50\%) täglich (Kategorie 7) bis mehrmals pro Woche (Kategorie 6) durchgeführt werden. Besonders Gesellschaftsspiele und Vorlesen gehören zu den Aktivitäten, die von fast allen Familien sehr häufig unternommen werden. Hier gibt es nur wenige Ausreißer. Bei den drei anderen Aktivitäten (Puzzeln, Singen und Geschichten erzählen) ist die Varianz der Antworten sehr viel größer und vereinzelt gibt es auch Familien, die die Aktivitäten nur seltener (2) oder sogar nie (1) mit ihrem Kind durchführen. Im Gegensatz dazu findet sich bei Familien, deren Betreuungsperson mindestens 12 Jahre (Abitur) die Schule besucht hat, wesentlich weniger Varianz. Besonders auffällig ist, dass fast alle Eltern ihrem Kind - nach eigenen Angaben - täglich vorlesen. Insgesamt zeigt Abbildung 3, dass die Aktivitäten in den Familien sehr häufig durchgeführt werden. In Familien mit dem höchsten Bildungsabschluss werden im Vergleich zu Familien mit Hauptschulabschluss signifikant häufiger Geschichten erzählt und es wird häufiger gesungen, beim Vergleich mit Familien mit Realschulabschluss finden sich signifikante Unterschiede beim Geschichtenerzählen, beim Spielen von Gesellschaftsspielen und beim Singen. Insgesamt ist die Varianz der Antworten bei Familien mit weniger Schulbildung größer. Auffällig ist, dass vor allem beim Puzzeln und bei Gesellschaftsspielen die Familien mit geringerer Schulbildung sogar angeben, dies häufiger zu tun als Familien mit höherer Schulbildung. Es stellt sich nun die Frage, ob diese Unterschiede mit Hilfe der elterlichen Freizeitaktivitäten erklärt werden können.

\section{Erklärung sozialer Unterschiede in den häuslichen Aktivitäten}

Die Analysen zur Erklärung von sozialen Unterschieden in den häuslichen Aktivitäten werden mit OLS-Regressionen durchgeführt. Tabelle 3 gibt einen Überblick über die Ergebnisse.

Zunächst werden in Modell 1 die soziale Herkunft und einige kindspezifische Variablen kontrolliert. Vor allem das Alter und das Geschlecht des Kindes wurden in der bisherigen Forschung als wichtige Einflussgrößen des Umfangs der häuslichen Aktivitäten diskutiert. Es zeigt sich, dass Betreuungspersonen mit Abitur im Vergleich zu Betreuungspersonen mit Hauptschulabschluss signifikant häufiger Aktivitäten mit ihren Kindern durchführen. Auch das Geschlecht wirkt sich signifikant auf die Aktivitäten aus: Mit Jungen werden weniger häufig Aktivitäten ausgeführt als mit Mädchen.

In Modell 2 werden die finanziellen Möglichkeiten der Eltern berücksichtigt, um auszuschließen, dass der Zusammenhang von Bildung und häuslichen Aktivitäten ausschließlich auf eine schlechte finanzielle Situation zurückzuführen ist. Diese Berücksichtigung erklärt kaum mehr Varianz der häuslichen Aktivitäten. Zudem üben die finanziellen Möglichkeiten keinen signifikanten Einfluss auf die hier gemessenen Aktivitäten aus und der Effekt des Bildungsabschlusses wird fast gar nicht beeinflusst. 
Tabelle 3: Einfluss von sozialer Herkunft und elterlichen Freizeitaktivitäten auf die häuslichen Aktivitäten

\begin{tabular}{|c|c|c|c|c|c|c|c|c|}
\hline \multirow{2}{*}{ Bildung $^{a}$} & \multicolumn{2}{|c|}{ Modell 1} & \multicolumn{2}{|c|}{ Modell 2} & \multicolumn{2}{|c|}{ Modell 3} & \multicolumn{2}{|c|}{ Modell 4} \\
\hline & & & & & & & & \\
\hline Realschule & 0,33 & $(0,20)$ & 0,32 & $(0,20)$ & 0,33 & $(0,20)+$ & 0,15 & $(0,17)$ \\
\hline (Fach-)Abitur & 0,41 & $(0,19)^{*}$ & 0,39 & $(0,20)^{*}$ & 0,39 & $(0,19)^{*}$ & 0,09 & $(0,16)$ \\
\hline Alter Zielkind in Monaten & $-0,02$ & $(0,01)$ & $-0,02$ & $(0,01)$ & $-0,02$ & $(0,01)$ & $-0,02$ & $(0,01)^{*}$ \\
\hline Geschlecht $^{\mathrm{b}}$ & $-0,22$ & $(0,09)^{*}$ & $-0,21$ & $(0,09)^{*}$ & $-0,21$ & $(0,09)^{*}$ & $-0,22$ & $(0,08)^{*}$ \\
\hline Äquivalenzeinkommen/1000 & & & 0,02 & $(0,03)$ & 0,03 & $(0,03)$ & 0,02 & $(0,03)$ \\
\hline Missingvariable: Einkommen & & & $-0,21$ & $(0,16)$ & 0,18 & $(0,15)$ & $-0,05$ & $(0,14)$ \\
\hline Erwerbstätigkeit $^{\mathrm{C}}$ & & & & & 0,31 & $(0,10)^{\star * *}$ & 0,32 & $(0,09)^{* * *}$ \\
\hline Anzahl Kinder im Haushalt & & & & & 0,14 & $(0,05)^{\star}$ & 0,16 & $(0,05)^{\star * *}$ \\
\hline Anzahl Kinderbücher/100 & & & & & 0,12 & $(0,05)^{*}$ & 0,03 & $(0,05)$ \\
\hline Kindergartenbesuchsdauer & & & & & 0,00 & $(0,00)$ & 0,00 & $(0,00)$ \\
\hline Elterliche Freizeitaktivitäten & & & & & & & 0,33 & $(0,05)^{\star * *}$ \\
\hline Konstante & 0,65 & $(0,64)$ & 0,79 & $(0,66)$ & 1,17 & $(0,65)$ & 1,61 & $(0,61)^{* *}$ \\
\hline $\mathrm{N}$ & \multicolumn{2}{|c|}{487} & \multicolumn{2}{|c|}{487} & \multicolumn{2}{|c|}{487} & \multicolumn{2}{|c|}{487} \\
\hline $\mathrm{R}^{2}$ & \multicolumn{2}{|c|}{0,0331} & \multicolumn{2}{|c|}{0,0365} & \multicolumn{2}{|c|}{0,0684} & \multicolumn{2}{|c|}{0,1556} \\
\hline
\end{tabular}

Anmerkung: Unstandardisierte Regressionskoeffizienten mit robusten Standardfehlern (in Klammern).

Referenzkategorien: a) Hauptschule, b) Mädchen, c) nicht Vollzeit erwerbstätig.

Signifikanzniveau: $+\mathrm{p} \leq 0,10 ; * \mathrm{p} \leq 0,05 ; * * \mathrm{p} \leq 0,01 ; * * * \mathrm{p} \leq 0,001$.

Quelle: Projekt „Erwerb von sprachlichen und kulturellen Kompetenzen von Migrantenkindern in der Vorschulzeit" (Welle 2), eigene Berechnungen.

In Modell 3 werden weitere Kontrollvariablen ergänzt, die die Möglichkeiten der Eltern, Aktivitäten durchzuführen, beeinflussen können. Dabei handelt es sich um Variablen, die zusätzlich die zeitlichen Möglichkeiten (Erwerbstätigkeit, Kindergartenbesuchsdauer), sowie das Vorhandensein von spezifischen Materialien (Kinderbücher) berücksichtigen. Es zeigt sich, dass auch die Kindergartenbesuchsdauer keinen signifikanten Einfluss ausübt. Im Gegensatz dazu wirken sich die Erwerbstätigkeit der Mutter und die Anzahl der Geschwister und der Kinderbücher signifikant positiv aus. Der Einfluss der Bildung wird durch die Berücksichtigung dieser Kontrollvariablen sogar etwas verstärkt - zumindest beim Vergleich von Eltern mit Hauptschulabschluss und Realschulabschluss. In Modell 4 soll nun überprüft werden, ob die hochkulturellen Freizeitaktivitäten der Eltern einen signifikanten Einfluss auf die häuslichen Eltern-Kind-Aktivitäten ausüben. Auch diese Annahme bestätigt sich und führt dazu, dass der Einfluss der Bildung verschwindet. Somit nimmt das hochkulturelle elterliche Freizeitverhalten eine vermittelnde Rolle beim Einfluss der sozialen Herkunft auf die häuslichen Aktivitäten ein.

\section{Diskussion}

Die vorgestellten Ergebnisse bestätigen zunächst, dass die häuslichen Aktivitäten verschiedene Kompetenzbereiche von (deutschen) Kindern signifikant beeinflussen. Im Anschluss daran konnte gezeigt werden, dass zwischen Familien signifikante Unterschiede in der Häufigkeit von spezifischen Aktivitäten in Abhängigkeit vom Bildungsabschluss der Betreuungsperson bestehen. Diese Unterschiede sind nicht allein auf die finanzielle 
und familiäre Situation der Familie zurückzuführen, sondern auch auf die hochkulturellen Freizeitaktivitäten der Eltern. Zudem konnte mit den multivariaten Analysen bestätigt werden, dass die zunächst vorhandenen Unterschiede zwischen Eltern mit unterschiedlichen Bildungsabschlüssen durch die elterlichen Freizeitaktivitäten erklärt werden können. Theoretisch deuten die Ergebnisse also in der Tat an, dass die Nutzenabwägung der Eltern bei den eigenen Freizeitaktivitäten auch im Rahmen der gemeinsamen Aktivitäten mit dem Kind stattfinden. Somit sind die zahlreichen empirischen Befunde, dass es soziale Unterschiede in den häuslichen Aktivitäten gibt, dadurch zu erklären, dass es weniger vom sozialen Hintergrund der Eltern als vielmehr von ihren nutzenorientierten Aktivitätspräferenzen abhängt. Nichtsdestotrotz hängen beide Arten der Aktivitäten von der Bildung der Eltern ab, wobei der Zusammenhang bei weitem nicht perfekt ist. Somit sprechen die Ergebnisse nicht dagegen, auch weiterhin die häuslichen Aktivitäten als ein mögliches Maß für SES-Unterschiede anzusehen. Jedoch soll der Beitrag darauf hinweisen, dass das häusliche Anregungsniveau eben nicht direkt von der Bildung der Eltern abhängt, zumindest nicht für sehr junge Kinder. Niklas und Schneider (2013) kommen zu dem Schluss, dass das HLE leichter zu verändern ist als die sozioökonomische Herkunft, jedoch zeigt sich in der hier vorliegenden Arbeit, dass es sich bei den häuslichen Aktivitäten um eine Projektion der eigenen Freizeitaktivitäten handelt, die in diesem Fall verändert werden müssten. Demnach müsste die Einschätzung der Eltern, die sie hochkulturellen Ressourcen beimessen, beeinflusst werden. Elternbildung sollte daher eher an der pädagogischen Orientierung und Überzeugung ansetzen.

Doch welche Schlussfolgerungen lassen sich daraus theoretisch und praktisch ziehen? Aus theoretischer Perspektive bestätigt sich, dass Eltern die Häufigkeit eigener Freizeitaktivitäten auch bei den Aktivitäten mit ihren Kindern umsetzen. Es bleibt jedoch weiterhin unklar, aus welchen Beweggründen dies der Fall ist. So sind sowohl traditionelle, wertrationale, zweckrationale, emotionale, habituelle oder noch andere Gründe denkbar. Bezogen auf die (frühe) soziale Bildungsungleichheit ist dieses Ergebnis aus praktischer Sicht sehr kritisch einzuordnen, da sich demnach Familien nur schwer darin beeinflussen lassen, wie häufig sie Aktivitäten mit ihren Kindern durchführen. Eltern sollten über den Wert der Bildung intensiv informiert werden und zusätzlich über Informationsmaterial oder gezielte Anleitung dazu motiviert werden, besonders entwicklungsförderliche Aktivitäten mit ihren Kindern zu unternehmen. Dies konnten Aram et al. (2013) auch unabhängig vom sozialen Hintergrund der Eltern nachweisen. In ihrer Studie zeigen sie, dass die gezielte Anleitung von Eltern die Förderung positiv beeinflusst. Gerade bei benachteiligten Familien fehlt vielleicht das Wissen zur Bedeutung dieser frühen Stimulation.

Daher sollte das Hauptaugenmerk auf andere Institutionen gelegt werden, die ähnlich wie die Eltern-Kind-Aktivitäten ebenfalls die kindlichen Kompetenzen positiv beeinflussen können. Die zentrale Institution in Deutschland, die dies leisten könnte, ist der Kindergarten. Die vorgestellten Analysen zeigen allerdings, dass die Kindergartenbesuchsdauer an sich keinen signifikanten Einfluss ausüben kann (vgl. Tabelle 2). Hier scheint es - gerade auch vor dem Hintergrund, dass inzwischen fast alle Kinder sehr frühzeitig einen Kindergarten besuchen - auf andere Faktoren des Kindergartens anzukommen (z.B. Qualität des Kindergartens, ethnische und soziale Zusammensetzung im Kindergarten). Diesbezüglich konnte gerade jüngst gezeigt werden, dass es zum Beispiel auch auf die ethnische und soziale Komposition im Kindergarten ankommt (vgl. Biedinger/Becker 2010). Ob aber der Kin- 
dergarten alleine in der Lage ist, mögliche familiäre Defizite auszugleichen, ist eine offene Frage. Hierzu fehlen bislang zumeist die notwendigen Daten.

Die Ergebnisse sind jedoch auch aus methodischer Sicht kritisch zu bewerten. Die vorliegende Arbeit misst die häuslichen Aktivitäten anhand sehr spezifischer Fragestellungen. Wünschenswert wären hier zusätzlich objektive Maße, so dass nicht nur die Häufigkeit, sondern auch die Qualität der Aktivitäten bzw. auch andere Faktoren gemessen werden könnten. Gerade auch für die Entwicklung der Kinder sind nicht nur diese spezifischen Aktivitäten, sondern auch der alltägliche Umgang zwischen Eltern und Kindern zentrale Determinanten für die sprachliche und kognitive Entwicklung. Weitere Angaben dazu und beispielsweise auch zum Erziehungsstil der Eltern wären erstrebenswert. Nichtsdestotrotz geben die Angaben zu den spezifischen Aktivitäten, so wie sie hier vorliegen, schon einen sehr guten Einblick in das häusliche Anregungsniveau, so dass davon auszugehen ist, das dieses Maß durchaus eine gute Approximation für die Erziehungsqualität und das damit verbundene Anregungsniveau der Familie ist.

Ein weiterer wichtiger Faktor, der die Möglichkeiten der Eltern zu gemeinsamen Aktivitäten beeinflussen kann, ist die tatsächlich vorhandene Zeit. Dies wurde in den Analysen auf verschiedene Weise berücksichtigt, nämlich die Zeit des Kindes über die Kindergartenbesuchsdauer und die Zeit der Betreuungsperson über die Erwerbstätigkeit. Diese beiden Maße sind jedoch ebenfalls nur sehr grobe Proxy für die tatsächliche Zeit, die der Betreuungsperson bleibt, um sich entwicklungsfördernd mit dem Kind zu beschäftigen. Hier wären - analog zu den internationalen Analysen - Zeittagebücher der Eltern wünschenswert, um genauer berücksichtigen zu können, wie lange welche Aktivität durchgeführt wird und wie viel Zeit die Eltern tatsächlich in diese Aktivitäten investieren.

Zusammenfassend gibt es zwar noch einige Indikatoren, von denen ein spezifischeres $\mathrm{Maß}$ wünschenswert wäre, es liegen aber dennoch gut verwendbare Variablen vor, so dass eine Fragestellung analysiert werden konnte, zu der bisher im deutschen Sprachraum nur wenige geeignete Datensätze zur Verfügung standen. Die Ergebnisse belegen, dass Eltern sich in der Tat darin unterscheiden, was sie mit ihren Kindern unternehmen. Und diese Unterschiede sind im besonderen Umfang darauf zurückzuführen, welche Aktivitäten die Eltern selbst häufig durchführen. Somit bestätigt sich die Annahme, dass Eltern mit ihrem Kind das unternehmen, was sie auch für sich selbst als positiv empfinden.

\section{Literatur}

Anders, Y., Rossbach, H.-G., Weinert, S., Ebert, S., Kuger, S. Simone Lehrl, S. \& von Maurice, J. (2012). Home and preschool learning environments and their relations to the development of early numeracy skills. Early Childhood Research Quarterly, 27, 2, S. 231-44.

Aram, D., Fine, Y. \& Ziv, M. (2013). Enhancing parent-child shared book reading interactions: Promoting references to the book's plot and socio-cognitive themes. Early Childhood Research Quarterly, 28,1, S.111-22.

Bandura, A. (1977). Social learning theory Englewood Cliffs, NJ: Prentice-Hall.

Becker, B. (2010a). The transfer of cultural knowledge in the early childhood. Social and ethnic disparities and the mediating role of familial activities. Europen Sociological Review 26, 1, S. 17-29.

Becker, B. (2010b). Who profits most from early parental investments? The effects of activities inside and outside the family on German and Turkish childrens's language development. Child Indicators Research 3, 1, S. 29-46. 
Becker, B. \& Biedinger, N. (2006). Ethnische Bildungsungleichheit zu Beginn der Schulzeit. Kölner Zeitschrift für Soziologie und Sozialpsychologie 58, 4, S. 660-684.

Becker, G. S. \& Tomes, N. (1976). Child endowments and the quantity and quality of children. The Journal of Political Economy, 84, 4, S.143-162.

Bianchi, S. M. (2000). Maternal employment and time with children: Dramatic change or surprising continuity? Demography, 37, 4, S. 401-414.

Biedinger, N. (2009a). Der Einfluss von elterlichen Investitionen auf die Entwicklung von deutschen und türkischen Kindern. Berliner Journal für Soziologie 19, 2, S. 268-294.

Biedinger, N. (2009b). Kinderarmut in Deutschland. Der Einfluss von relativer Einkommensarmut auf die kognitive sprachliche und behavioristische Entwicklung von 3- bis 4-jährigen Kindern. Zeitschrift für Soziologie der Erziehung und Sozialisation 29, 2, S. 197-214.

Biedinger, N. \& Becker, B. (2010). Frühe ethnische Bildungsungleichheit. Der Einfluss des Kindergartenbesuchs auf die Schulfähigkeit. In: Becker, B. \& Reimer, D. (Hrsg.), Vom Kindergarten bis zur Hochschule. Die Generierung von ethnischen und sozialen Disparitäten in der Bildungsbiographie. Wiesbaden: VS Verlag für Sozialwissenschaften, S. 49-80.

Boudon, R. (1974). Education, opportunity, and social inequality. Changing prospects in western society New York: Wiley \& Sons.

Bourdieu, Pi. (1976). Soziale Ungleichheit - Strukturen und Prozesse sozialer Schichtung. In; Hörning, K. H. (Hrsg.), Kulturelle Reproduktion und soziale Reproduktion. Darmstadt: Hermann Luchterhand Verlag, S. 223-231.

Bradley, R. H., Mundfrom, D.J.,Whiteside, L., Caldwell, B. M., Casey, P. H., Kirby, R. S. \& Hansen, S. (1994). A reexamination of the association between HOME scores and income. Nursing Research, 43,5 , S. $260-266$.

Bradley, R. \& Robert Corwyn, R. (2005). Caring for children around the world: A view from HOME. International Journal of Behavioral Development, 29, 6, S. 468-478.

Bryant, W. K. \& Zick, C. D. (1996a). Are we investing less in the next generation? Historical trends in time spent caring for children. Journal of Family and Economic Issues, 17, 3/4, S. 365-392.

Bryant, W. K. \& und Cathleen D. Zick, C. D. (1996b). An examination of parent-child shared time. Journal of Marriage and the Family, 58, 1, S. 227-37.

Bus, Adrian G., Ijzendoorn, M. H. \& Pellegrini, A. D. (1995). Joint book reading makes for success in learning to read. A meta-analysis on intergenerational transmission of literacy. Review of Educational Research, 65, 1, S. 1-21.

Conger, K. J., Rueter, M. A. \& Conger, R. D. (2000). Negotiating adolescence in times of social change. In: Crocket, L. J. \& Silbereisen, R. K. (Hrsg.), The role of economic pressure in the lives of parents and their adolescents: The family stress model. Cambridge: Cambridge University Press, S. 201223.

Craig, L. (2006). Parental education, Time in paid work and time with children: An Australian time-diary analysis. The British Journal of Sociology, 57, 4, S. 553-575.

Craig, L. (2007). How employed mothers in Australia find time for both market work and childcare. Journal of Family and Economic Issues, 28, 1, S. 69-87.

Denton, K. L., Reaney, L. M. \& West, J. (2001). Papers from the Early Childhood Longitudinal Studies Program presented at the 2001 AERA and SRCD Meetings. In: West, J. (Hrsg.), Home educational activities, literacy resources und kindergartners' reading knowledge and skills. Washington, D.C.: National Center for Education Statistics.

Diewald, M. \& Schupp, J. (2004). Generation und Ungleichheit. In: Szydlik, M. (Hrsg.), Soziale Herkunft, Beziehung zu den Eltern und das kulturelle und soziale Kapital von Jugendlichen, Wiesbaden: VS Verlag für Sozialwissenschaften, S. 104-127.

Downer, J. T. \& Pianta, R. C. (2006). Academic and cognitive functioning in first grade: Associations with earlier home and child care predictors and with concurrent home and classroom experiences. School Psychology Review, 35, 1, S. 11-30.

Esser, H. (1996). Die Definition der Situation. Kölner Zeitschrift für Soziologie und Sozialpsychologie, 48,1, S. $1-34$. 
Farran, D. C. \& Ramey, C. T. (1980). Social class differences in dyadic involvement during infancy. Child Development, 51, 1, S. 254-257.

Garrett, P., Ng'andu, N. \& Ferron, J. (1994). Poverty experiences of young children and the quality of their home environments. Child Development, 65, 2, S. 331-45.

Grundmann, M. (2001). Milieuspezifische Einflüsse familialer Sozialisation auf die kognitive Entwicklung und den Bildungserfolg. In: Klocke, A. \& Hurrelmann, K. (Hrsg.), Kinder und Jugendliche in Armut. Umfang, Auswirkungen und Konsequenzen. Wiesbaden: Westdeutscher Verlag, S. 161-182.

Hart, B. \& Risley, T. R. (1995). Meaningful differences in the everyday experience of young American children Baltimore: Paul H. Brookes Publishing Co.

Hashima, P.Y. \& Amato, P. R. (1994). Poverty, social support, and parental behavior. Child Development, 65, 2, S. 394-403.

Hill, C. R. \& Stafford, F. P. (1980). Parental care of children: Time diary estimates of quantity, predictability, and variety. The Journal of Human Resources, 15, 2, S. 219-239.

Hoffa, E. (20039. Socioeconomic status, parenting, and child development. In: Bornstein, M. H. \& Bradley, R. H. (Hrsg.), Causes and consequences of SES-related differences in parent-to-child speech. Mahwah: Lawrence Erlbaum Associates, S. 147-160.

Hofferth, S, L. (2001). Women's employment in a comparative perspective. In: Van Dijk, L. \& Van der Lippe, T. (Hrsg.), Women's employment and care of children in the United States. New York: Aldine de Gruyter, S, 151-174.

Kaufman, A. S. \& N. L. Kaufman, N. L. (1994). Kaufman Assessment Battery for Children. K-ABC Frankfurt: Swets Test Service.

Kitterød, R. H. (2002). Mothers' housework and childcare: Growing similarities or stable inequalities? Acta Sociologica, 45, 2, S.127-49.

Kohn, M. L. (1969). Class and conformity. A study in values. Homewood: The Dorsey Press.

Kroneberg, C. (2007). Wertrationalität und das Modell der Frame-Selektion. Kölner Zeitschrift für Soziologie und Sozialpsychologie, 59, 2, S. 215-239.

Künzler, J., Walter, W., Reichart, E. \& Pfister, G. (2001). Gender division of labor in unified Germany. http://www.politikwissenschaft.uni-wuerzburg.de/fileadmin/06060101/na_rep.pdf

Lareau, A. (2002). Invisible inequality: Social class and childrearing in black families and white families. American Sociological Review, 67, 5, S. 747-76.

Lehrl, S., Ebert, S., Roßbach, H.-G. \& Weinert, S. (2012). Die Bedeutung der familiären Lernumwelt für Vorläufer schriftsprachlicher Kompetenzen im Vorschulalter. Zeitschrift für Familienforschung/ Journal of Family Research, 24, 2, S. 115-133.

Luster, T. \& Dubow, E. (1992). Home environment und maternal intelligence as predictors of verbal intelligence: A comparison of preschool and school-age children. Merrill-Palmer Quarterly, 38, 2, S. 151-175.

Melchers, P. \& Preuß, U. (2001). Kaufman-Assessment Battery for Children. Deutschsprachige Fassung. Leiden: PITS.

Melchers, P. \& Preuß, U. (2003). K-ABC. Kaufman-Assessment Battery for Children. Deutschsprachige Fassung. Interpretationshandbuch Frankfurt am Main: Swets \& Zeitlinger.

Melhuish, E. C., Phan, M. B., Sylva, K., Pam Sammons, Siraj-Blatchford, I. \& Taggart, B. (2008). Effects of the home learning environment and preschool center experience upon literacy and numeracy development in early primary school. Journal of Social Issues, 64, 1, S. 95-114.

Niklas, F. \& Schneider, W. (2010). Der Zusammenhang von familiärer Lernumwelt mit schulrelevanten Kompetenzen im Vorschulalter. Zeitschrift für Soziologie der Erziehung und Sozialisation, 30, 2, S. 149-65.

Niklas, F. \& Schneider, W. (2012). Einfluss von „Home Numeracy Environment“" auf die mathematische Kompetenzentwicklung vom Vorschulalter bis Ende des 1. Schuljahres. Zeitschrift für Familienforschung/Journal of Family Research, 24, 2, S. 134-147.

Niklas, F. \& Schneider, W. (2013). Home literacy environment and the beginning of reading and spelling. Contemporary Educational Psychology, 38, 1, S. 40-50.

Pauen, S., Janna Pahnke, J. \& Valentiner, I. (2007), Erfassung kognitiver Kompetenzen im Vorschul- bis Jugendalter: Intelligenz, Sprache und schulische Fertigkeiten. http://www.diw.de/documents/ publikationen/73/diw_01.c.57321.de/rn20.pdf 
Raviv, T., Kessenich, M. \& Morrison, F. J. (2004). A mediational model of the association between socioeconomic status and three-year-old language abilities: The role of parenting factors. Early Childhood Research Quarterly, 19, 4, S. 528-547.

Rössel, J. \& Beckert-Zieglschmid, C. (2002). Die Reproduktion kulturellen Kapitals. Zeitschrift für Soziologie, 31, 6, S. 497-513.

Sandberg, J. F. \& Hofferth, S. L. (2001). Changes in children's time with parents: United States, 19811997. Demography, 38, 1, S. 423-36.

Schöler, H., Guggenmos, J., Hasselbach, J. \& Iseke, A (2005). Sprachliche Leistungen in der Einschulungsuntersuchung. Ein Vergleich der Jahrgänge 1999 bis 2004 in der Stadt Münster. Heidelberg: Pädagogische Hochschule, Institut für Sonderpädagogik, Abteilung Psychologie in Sonderpädagogischen Handlungsfeldern

Sénéchal, M. \& Young, L. (2008). The effect of family literacy interventions on children's acquisition of reading from kindergarten to grade 3: A meta-analytic review. Review of Educational Research, 78, 4, S. 880-907.

Totsika, V. und Kathy Sylva, K. (2004). The Home Observation for Measurement of the Environment revisited. Child and Adolescent Mental Health, 9, 25-35.

van Steensel, R. (2006). Relations between socio-cultural factors, the home literacy environment and children's literacy development in the first years of primary education. Journal of Research in Reading, 29, 4, S. 367-82.

Walter, W. \& Künzler, J. (2002). Elternschaft heute. Gesellschaftliche Rahmenbedingungen und individuelle Gestaltungsaufgaben. In: Schneider, N. F. \& Matthias-Beck, H. (Hrsg.), Parentales Engagement. Mütter und Väter im Vergleich. Opladen: Leske + Budrich, S. 95-119.

Weber, M. (1980). Wirtschaft und Gesellschaft. Grundriß der verstehenden Soziologie. Tübingen: Mohr Siebeck.

Weinert, S., Ebert, S., Lockl, K. \& Kuger, S. (2012). Disparitäten im Wortschatzerwerb: Zum Einfluss des Arbeitsgedächtnisses und der Anregungsqualität in Kindergarten und Familie auf den Erwerb lexikalischen Wissens. Unterrichtswissenschaft, 40, 1, S. 4-25.

Yarosz, D. \& Barnett, W. (2001). Who reads to young children? Identifying predictors of family reading activities. Reading Psychology, 22, 1, S. 67-81.

Yeung, J., Sandberg, J. F., Davis-Kean, P. \& Hofferth, S. (2001). Children's time with fathers in intact families. Journal of Marriage and the Family. 63, 1, S. 136-154.

Zick, C. D. \& Bryant, W. K. (1996). A new look at parents' time spent in child care: Primary and secondary time use. Social Science Research, 25, 3, S. 260-280.

Zick, C. D., Bryant, W. K. \& Österbacka, E. (2001). Mothers' employment, parental involvement, and the implications for intermediate child putcomes. Social Science Research, 30, 1, S. 25-49.

Eingereicht am/Submitted on: 05.11.2012

Angenommen am/Accepted on: 07.08.2013

Anschrift der Autorin/Address of the author:

Dr. Nicole Biedinger

GESIS - Leibniz-Institut für Sozialwissenschaften

Postfach 122155

68072 Mannheim

Deutschland/Germany

E-Mail: Nicole.Biedinger@gesis.org 